Lehmann, B.A., Bos, A.E.R., Rijken, M., Cardol, M., Peters, G.J.Y., Kok, G., Curfs, L.M.G. Ageingh with an intellectual disability: the impact of personal resources on well-being. Journal of Intellectual Disability Research: 2013, 57(11), 1068-1078

\begin{tabular}{|l|l|}
\hline $\begin{array}{l}\text { Postprint } \\
\text { Version }\end{array}$ & 1.0 \\
\hline Journal website & http://onlinelibrary.wiley.com/doi/10.1111/j.1365-2788.2012.01607.x/pdf \\
\hline Pubmed link & http://www.ncbi.nlm.nih.gov/pubmed/22974110 \\
\hline DOI & $10.1111 / j .1365-2788.2012 .01607 . x$ \\
\hline
\end{tabular}

This is a NIVEL certified Post Print, more info at http://www.nivel.eu

\title{
Ageing with an intellectual disability: the impact of personal resources on well-being
}

\author{
B. A. LehMANN ${ }^{1, *}$, A. E. R. Bos ${ }^{2}$, M. RIJKEN ${ }^{3}$, M. CARDOL ${ }^{4}$, G.-J. Y. PETERs ${ }^{2}$, G. KOK ${ }^{5}$, L. \\ M. G. CURFS ${ }^{6}$
}

\begin{abstract}
Background The population of ageing people with mild and moderate intellectual disabilities (ID) is growing rapidly. This study examines how personal resources (physical health, mental health and social networks) impact the well-being of ageing people with ID.

Methods Longitudinal survey data on 667 people with a mild or moderate ID were acquired via interviews in 2006 and 2010. Indicators of personal resources (physical health, mental health and social networks) were assessed, as were indicators of well-being (satisfaction with life, happiness and loneliness). Additionally, data on background characteristics and autonomy were gathered.

Results The results show that age is positively related to decreased mobility and auditory disabilities and negatively related to independent living, autonomy in how one spends one's leisure time and autonomy in decision-making. Longitudinal analyses demonstrated that, with the exception of health that deteriorated, and social satisfaction that improved, almost all variables remained stable over the 4-year period. Further, good physical health in 2006 predicted happiness in 2010.

Conclusion Despite the fact that age is associated with poorer physical and mental health and a smaller social network, this study showed that older people with ID have relatively high levels of well-being. Findings are discussed in the light of coping with ageing and impact of life events.
\end{abstract}

\section{INTRODUCTION}

Participation in community activities contributes to well-being (Losier et al. 1993). Unfortunately, people with intellectual disabilities (ID) participate less in community activities than people without disabilities (De Klerk 2002; Dusseljee et al. 2011). This may be due to a lack of resources such as health, cognitive skills, certain personality characteristics, characteristics of the physical and social environment, and finances (Diener \& Fujita 1995). In this study, we focus on personal resources, namely physical health, mental health and social networks, and their impact on wellbeing. This is especially important in ageing people with ID for two reasons. First, 
Lehmann, B.A., Bos, A.E.R., Rijken, M., Cardol, M., Peters, G.J.Y., Kok, G., Curfs, L.M.G. Ageingh with an intellectual disability: the impact of personal resources on well-being. Journal of Intellectual Disability Research: 2013, 57(11), 1068-1078

people with ID tend to, in general, have poorer physical and mental health, and smaller social networks, and are therefore less able to use these resources to gain well-being than the general population (Ormel et al. 1996). Second, regardless of the population, physical and mental health problems are known to increase and social network size to decrease with age (Zantinge et al. 2011). In this article, we consider people with ID aged 50 and older to be 'older' or 'ageing' as research has shown that people with ID at 50 years of age are comparably vulnerable to people aged 65 in the general population (Evenhuis 2011).

The aim of the study reported here was to, in a sample of people with ID, first, replicate findings that have shown an association between ageing and physical health, mental health and social network size and, second, investigate the relationship between these personal resources and well-being of ageing people with ID. In the following paragraph we will discuss current knowledge on physical and mental health, as well as social networks of ageing people with ID.

\section{Physical health, mental health and social networks}

Nowadays, people with ID experience an increased life expectancy similar to the general population, as a result of improved living conditions and a better medical care (Thorpe et al. 2001). Studies on the mortality of people with ID show that cardiovascular, respiratory diseases and cancer are the most common causes of death, which is comparable to the general population (Janicki et al. 1999). What differs is the fact that people with ID are considerably more likely to experience several health problems across their lifespan (van Schrojenstein Lantman-de Valk et al. 2000; Cardol et al. 2012). Also, people with ID have been found to show characteristics of premature ageing (Carmeli et al. 2005). In fact, physical health problems such as osteoporosis, thyroid disorders, non-ischemic heart diseases, epilepsy and diseases of the skin are increasingly present in people with ID around middle age (Bittles et al. 2002; Jansen et al. 2004). Hearing and visual impairments also show a sharp increase after age 50 (Evenhuis et al. 2001).

In addition to physical health problems, people with ID also experience mental health issues. Psychopathology is prominent in people with ID (Thorpe et al. 2001; Kearney \& Healy 2011) and a number of studies have demonstrated that psychopathology increases with age (Thorpe et al. 2001; Carmeli et al. 2005). In general, the focus has been on dementia and this is not surprising given its relevance among ageing people in general and ageing people with ID in particular (McCallion \& McCarron 2004). In fact, previous research has shown that dementia not only occurs more often in people with ID but that it also tends to occur at a younger age than in the general population (McCarron \& Lawlor 2003).

Further, the social networks of people with ID have been reported to be restricted and to primarily consist of family members, health care staff and other people with ID (Forrester-Jones et al. 2006; Dusseljee et al. 2011). The social networks of people with ID may also include neighbours for those living in communities and not in facilities (van Alphen et al. 2009). However, research has shown that living in a community and having neighbours does not guarantee contact to neighbours (van Alphen et al. 2009; Dijker et al. 2011). Generally, parents assume the most prominent role in the social networks of people with ID but as people with ID age, so do their parents and their chronic illnesses and eventual death can result in a significant loss of social support to people with ID (Bigby 1997). Ageing people with 
Lehmann, B.A., Bos, A.E.R., Rijken, M., Cardol, M., Peters, G.J.Y., Kok, G., Curfs, L.M.G. Ageingh with an intellectual disability: the impact of personal resources on well-being. Journal of Intellectual Disability Research: 2013, 57(11), 1068-1078

ID are thus likely to have too few social relationships and may therefore suffer from loneliness (Duvdevany \& Arar 2004).

\section{The present study}

Taking a closer look at the personal resources of ageing people with ID, namely their physical and mental health and their social networks, is imperative, especially given the likelihood that these resources are often limited to start with and tend to decrease with age. Consequently, this study set out to investigate the following research questions: (1) is age related to the physical and mental health, and the social network size, of people with ID as has been documented in previous research? (2) how stable over time are the personal resources older people with ID have at their disposal? and (3) in people with ID aged 50 and older, which personal resources are associated with well-being 4 years later?

Our hypotheses are as follows: (1) advanced age will be related to decreased physical and mental health and a smaller social network; (2) personal resources of older people with ID will decrease over the 4-year period; and (3) decreased physical and mental health and a smaller social network will be negatively related to well-being in people with ID aged 50 and older.

\section{METHOD}

\section{Participants}

The present cohort study is part of a national prospective panel study called 'Panel Living Together', which started in 2006 in the Netherlands. To recruit participants, the total population of institutions $(n=167)$ that provided living arrangements for people with ID in the Netherlands at that time were contacted in writing, of which 55 actually assisted. Also, a random sample of general practitioners (GP; $n=89$ ) identified people with ID by means of a checklist developed by Van Schrojenstein Lantman-de Valk et al. (2004) in order to reach people with ID that do not use services for people with ID. Inclusion criteria for this study were a diagnosis of a mild or moderate ID as defined by the American Association on Intellectual and Developmental Disabilities (Schalock et al. 2010), participants had to be older than 15 years of age and were expected to live for at least another 6 months according to the caregiver involved. People with ID that were identified by a facility or GP and that met the inclusion criteria were sent an information package containing a letter with pictograms and a CD with information concerning the 'Panel Living Together' in the form of a dialogue. Potential participants or their representatives (in the event that participants were younger than 18 or unable to understand the study's purpose) were asked to sign and return an informed consent. In 2010, institutions and GPs were contacted a second time to obtain new panel members and to compensate for earlier dropouts, which resulted in 109 new members. These acquisition procedures yielded a total of 667 people (369 men and 298 women) with a mild or moderate ID or representatives of people with ID.

\section{Procedure}

In this study, we used data collected via oral interviews with the panel members with ID in the autumn of 2006 and the autumn of 2010. In order to make the interviews as 
Lehmann, B.A., Bos, A.E.R., Rijken, M., Cardol, M., Peters, G.J.Y., Kok, G., Curfs, L.M.G. Ageing with an intellectual disability: the impact of personal resources on well-being. Journal of Intellectual Disability Research: 2013, 57(11), 1068-1078

uniform as possible, interviewers received oral instructions as well as a training on how to communicate with people with ID. The interviews were conducted in the participants' homes or residential facilities. The interviews lasted a maximum of an hour. A total of $45 \%$ of the participants were interviewed in the presence of a third person. For a more detailed description of the participants and procedure, see Cardol et al. (2006).

\section{Measurements}

People with ID deal best with particular questions in oral interviews (Finlay \& Lyons 2001; Lloyd et al. 2006). As such, and taking into consideration participants' ability to communicate and verbalise their situation, most questions were measured dichotomously.

\section{Personal resources}

Perceived physical and mental health was measured with three dichotomous items: (1) physical health: do you feel healthy? (yes/no); (2) mobility: can you walk $400 \mathrm{~m}$ at once (one time around a soccer field) without standing still (if necessary with a walker)? (yes/no); and (3) presence of disabilities (indicating which are present and number): which of the following health problems are present? (none, visual impairments, auditory impairments, motor/physiological problems, communication deficits, autism, behavioural problems, psychiatric problems, addiction, dementia, epilepsy) (yes/no).

Social networks were measured with three dichotomous items: (1) social satisfaction: are you talking to and meeting enough people? (yes/no); (2) partner: do you have a partner/a steady boyfriend or girlfriend? (yes/no), (2.1) partner with ID: does your partner have ID? (yes/no); and (3) children: do you have children? (yes/no), (3.1) if so, how many children do you have? (number).

Autonomy was considered an additional contributor to well-being. Therefore, perceived autonomy was measured with two ordinal variables: (1) can you decide how to spend your free time? (often/sometimes/rarely to never); and (2) can you make your own choices? (often/sometimes/rarely to never).

\section{Outcome measures}

Indicators of well-being were assessed by three dichotomous items: (1) satisfaction with life (cognitive component): are you satisfied with your life? (yes/no); (2) happiness (affective component, measured only in 2010): do you feel happy most of the time? (yes/no); and (3) loneliness (social component): do you sometimes feel lonely? (yes/no).

\section{Background variables}

Information regarding gender, age, severity of the ID (mild or moderate), accommodation type (in the community or in an institutional setting) and additional mental or physical health conditions were provided by GPs or care providers.

\section{Analyses}

In our cross-sectional analyses whereby we investigated if age is related to physical and mental health and social networks, we used t-tests for dichotomous variables, 
Lehmann, B.A., Bos, A.E.R., Rijken, M., Cardol, M., Peters, G.J.Y., Kok, G., Curfs, L.M.G. Ageing with an intellectual disability: the impact of personal resources on well-being. Journal of Intellectual Disability Research: 2013, 57(11), 1068-1078

ANOVA for ordinal variables and Pearson correlations for interval variables. In our longitudinal analyses, we selected participants aged 50 years and older and compared the data acquired in 2006 with the data acquired in 2010 using the McNemar test to examine variable stability over time and the $\chi 2$-test to examine associations between variables in 2006 and 2010. We considered significance not only at the customary level of $\alpha=0.05$ but also at a more conservative level $(\alpha=0.01)$ because conducting a large number of statistical tests increases the likelihood of type 1 errors. In order to alleviate problems associated with null hypothesis testing (e.g. Cohen 1990; Kirk 2007), we computed effect sizes for all associations, and because of the large number of analyses we report, we report primarily these effect sizes and the significance levels, including the statistics and degrees of freedom in Appendix 1. We obtained effect sizes by computing odds ratios for associations between two dichotomous variables, Cramèr's $\mathrm{V}$ for associations between a dichotomous and an interval variable, $\eta 2$ for associations of an ordinal and an interval variable, and Cohen's d for associations between a dichotomous and an interval variable. In addition to the quantitative effect sizes, we used qualitative labels (Cohen 1988; Rosenthal 1996) to facilitate interpretation of the large number of outcomes (see Table 1). We considered associations to be relevant when they were: (a) significant at the $\alpha=0.05$ level with at least a medium effect size; or (b) significant at the $\alpha=0.01$ level with at least a small effect size. Associations that just failed to meet these criteria were considered marginally relevant. Note that it is necessary to evaluate P-value and effect size simultaneously, as they are not deterministically dependent; a given P-value is a function of both an effect size and a sample size.

\section{[TABLE 1]}

\section{RESULTS}

The absolute and relative frequencies of the dichotomous variables that were measured in 2006 and 2010 are shown in Table 2. The mean age of the entire sample $(n=667)$ was 47 years. Among participants aged 50 and older $(n=284,43 \%)$, the mean age was 61 years. Roughly half of the participants were male [ $\mathrm{n}=369(55 \%)$ of the total sample and $n=145$ (51\%) of the subsample]. Both in the total sample and in the subsample of older people with ID, 61\% had a mild ID ( $n=407)$ and 39\% had a moderate ID $(n=260)$. In terms of autonomy in how one spends one's leisure time (derived from the 2010 data), 11 participants (2\%) indicated rarely being able to decide how to spend their leisure time, 66 participants (14\%) indicated sometimes and 393 participants (84\%) said they were often able to decide how to spend their leisure time. With respect to autonomy in decision-making, 29 participants (6\%) said they rarely make their own choices, 92 participants (19\%) sometimes make their own choices and 360 participants (75\%) often make their own choices. The descriptive statistics for the continuous variables (age, number of children and number of disabilities) are shown in Table 3.

[TABLE 2- 3]

\section{Cross-sectional analyses}


Lehmann, B.A., Bos, A.E.R., Rijken, M., Cardol, M., Peters, G.J.Y., Kok, G., Curfs, L.M.G. Ageing with an intellectual disability: the impact of personal resources on well-being. Journal of Intellectual Disability Research: 2013, 57(11), 1068-1078

The outcomes of the cross-sectional analyses are shown in Table 4. In these analyses, age was related to decreased mobility and more auditory disabilities (medium effect sizes). Age was also negatively associated with independent living, autonomy in how one spends one's leisure time and autonomy in decision-making (small effect sizes). In terms of marginally relevant associations, we found age to be associated with poorer physical health, more visual impairment and more psychiatric problems. At the same time, age was also related to greater social satisfaction, more satisfaction with life and less loneliness, in addition to a lower frequency of autism and fewer communication deficits.

\section{[TABLE 4]}

\section{Longitudinal analysis}

The outcomes of the longitudinal analyses are shown in Table 5. McNemar tests, which assessed the null hypothesis that relative frequencies do not change over time, showed that only physical health and social satisfaction changed between 2006 and 2010. The proportion of participants reporting good physical health decreased from $87 \%$ to $83 \%$ between 2006 and 2010, while the proportion of participants reporting relatively high levels of social satisfaction increased from $72 \%$ to $86 \%$. As for predictors of well-being in 2010, expressed positively as satisfaction with life and happiness and negatively as loneliness (research question 3), we found no relevant associations between any of the variables measured in 2006 and satisfaction with life or loneliness in 2010, but we did find a marginally relevant association indicating that participants reporting better physical health in 2006 were less lonely in 2010. What we did find was that participants reporting better physical health in 2006 were also happier in 2010.

\section{[TABLE 5]}

\section{DISCUSSION}

The present study investigated, in people with a mild or moderate ID, how personal resources in the form of physical and mental health as well as social network size are related to well-being expressed positively as satisfaction with life and happiness and negatively as loneliness. The results showed that in 2010, age was negatively related to independent living, autonomy in deciding how to spend one's leisure time and autonomy in decision-making. This clearly indicates that older people with ID tend to have less autonomy than younger people with ID. Moreover, we found ageing to be associated with less mobility and more auditory disabilities, which is in line with previous research (van Schrojenstein Lantman-de Valk et al. 2000). We also found marginally relevant associations between age, on the one hand, and poorer physical health, more visual impairment and more psychiatric disabilities, on the other. Additionally, communication deficits and autism appeared to be more frequently related to younger age.

At the same time, we found age to be related to more satisfaction with life, greater satisfaction with the social contacts one has and less loneliness. This was unexpected and not in line with our hypothesis but these results are in line with previous research 
Lehmann, B.A., Bos, A.E.R., Rijken, M., Cardol, M., Peters, G.J.Y., Kok, G., Curfs, L.M.G. Ageingh with an intellectual disability: the impact of personal resources on well-being. Journal of Intellectual Disability Research: 2013, 57(11), 1068-1078

on ageing among the general population (Smith et al. 2002; Zantinge et al. 2011). For example, Smith et al. (2002) showed that, despite poorer health, older people maintain a relatively stable psychological quality of life. One possible explanation is that this is due to changes in older people's needs and expectations about, for example, the number of friends they have (Zantinge et al. 2011). Another explanation is provided by Frieswijk et al. (2004) who suggested that ageing people adjust the criteria by which they determine if they are satisfied with their lives by using downward social comparisons. Furthermore, although age was related to less autonomy, this appeared to not affect satisfaction with life, happiness or loneliness. Future research should investigate the concept of autonomy in the lives of ageing people with ID in greater detail.

The longitudinal investigation of the data further revealed that most physical and mental health indicators, as well as indicators of well-being, remained stable over the 4-year period. Exceptions were that perceived health decreased over the 4-year period while satisfaction with social contacts increased. Poorer perceived health of participants aged 50 over the 4-year period was not unexpected and may indicate that physical vulnerability becomes increasingly visible from 50 years of age on, as suggested by Evenhuis (2011). Increases in social satisfaction may again be the result of changing expectations and needs as previously posited by Zantinge et al. (2011). Another potential explanation is that older people with ID cope better than their younger counterparts. Unfortunately, we did not collect data on coping strategies employed by people with ID but do recommend that future research explore coping strategies.

We also found that indicators of personal resources (physical health, mental health, social network size) were hardly predictive of well-being 4 years later. The only associations found were that older participants who perceived themselves to be relatively healthy in 2006 were also happier in 2010 and that perceived health in 2006 may also be predictive of less loneliness in 2010 but this finding was only marginally relevant. One explanation for the fact that few variables measured in 2006 predicted well-being in 2010 can be found in the work of Finlay \& Lyons (2001) who contend that questions that require one to imagine time are difficult for people with ID. The lack of prediction demonstrated in our study may therefore be because participants' answers to the questions reflected what they felt at that moment rather than a stable state. It is also possible that variables other than the ones we measured are predictive of well-being in older people with ID. For example, family support may be an important contributor to well-being (Forrester-Jones et al. 2006). Unfortunately, we did not collect data about the presence or absence of parents in the lives of the participants or which coping strategies participants use. Likewise, other important life events (e.g. relocation, development of a chronic disease), could have been additionally related to the well-being of ageing people with ID.

\section{Strengths and limitations}

Our study has a number of strengths, the first of which is the longitudinal nature of the study. This is particularly important given that current need to monitor the development of the rapidly growing population of ageing people with ID (McCallion \& McCarron 2004). Second, this study comprised a large sample $(n=667)$ from 55 institutions and 89 GPs. Unfortunately, there are no concrete information about why some institutions and GPs were not willing to help recruit participants for the panel. 
Lehmann, B.A., Bos, A.E.R., Rijken, M., Cardol, M., Peters, G.J.Y., Kok, G., Curfs, L.M.G. Ageingh with an intellectual disability: the impact of personal resources on well-being. Journal of Intellectual Disability Research: 2013, 57(11), 1068-1078

Therefore, an unintended selection bias cannot be ruled out. Third, the data were gathered nationwide rather than in one location. Fourth, this study is one of very few panel studies to explore the physical and mental health, social networks and wellbeing in such a large sample of people with ID.

In addition to the strengths, this study also has some limitations. First, most of the items were dichotomous as asking people with ID to answer questions is not always easy. Many people with ID have difficulties conveying feelings and situations, and imagining time (Lloyd et al. 2006). Nonetheless, there is a need for standardised measures and this poses a considerable challenge to researchers. Second, some associations were barely significant and had weak effect sizes and should therefore be interpreted with caution. These marginally significant results, however, provide clear guidelines for future research. In addition, our large sample size gives us sufficient power to conclude with considerable confidence that non-significant associations indeed do not exist. Future research should aim to replicate these findings. Lastly, it is possible that 4 years is not long enough to find significant changes in the well-being of people with ID. Therefore, a follow-up study later in time could reveal important changes, especially in older participants.

\section{Implications for future research}

Given that few variables measured in 2006 predicted well-being in 2010, we recommend that future research include other potentially relevant variables such as the family composition of ageing people with ID and life events that may impact well-being (e.g. relocation, the development of chronic disease). Furthermore, we suggest that future research includes questions on the coping strategies people with ID employ in dealing with increasing age. Finally, we recommend comparing ageing people with ID across countries as the health care system may play a role in the wellbeing of ageing people with ID. For example, the quality of care provided may explain why well-being stays remained despite decreases in physical and mental health.

\section{REFERENCES}

Bigby C. (1997) Parental substitutes? The role of siblings in the lives of older people with intellectual disability. Journal of Gerontological SocialWork 29, 3-21.

Bittles A. H., Petterson B. A., Sullivan S. G., Hussain R., Glasson E. J. \& Montgomery P. D. (2002) The influence of intellectual disability on life expectancy. Journal of Gerontology: Medical Sciences 57A, M470-M472.

Cardol M., Speet M. \& Rijken M. (2006) Anders of toch niet? Deelname aan de samenleving van mensen met een verstandelijke beperking (Different or Not? Participation in the Social Interaction of People with an Intellectual Disability). NIVEL, Utrecht.

Cardol M., Rijken M. \& van Schrojenstein Lantman-de Valk H. (2012) People with mild to moderate intellectual disability talking about their diabetes and how they manage. Journal of Intellectual Disability Research 56, 351-60.

Carmeli E., Zinger-Vaknin T., Morad M. \& Merrick J. (2005) Can physical training have an effect on wellbeing in adults with mild intellectual disability? Mechanisms of Ageing and Development 126, 299-304.

Cohen J. (1988) Statistical Power Analysis for the Behavioral Sciences, 2nd edn. Lawrence Erlbaum, Hillsdale, NJ.

Cohen J. (1990) Things I have learned (so far). American Psychologist 45, 1304-12.

De Klerk M. M.Y. (ed.) (2002) Rapportage gehandicapten 2002. Maatschappelijke positie van mensen met lichamelijke beperkingen of verstandelijke handicaps (Report 
Lehmann, B.A., Bos, A.E.R., Rijken, M., Cardol, M., Peters, G.J.Y., Kok, G., Curfs, L.M.G. Ageing with an intellectual disability: the impact of personal resources on well-being. Journal of Intellectual Disability Research: 2013, 57(11), 1068-1078

Handicapped People 2002. Societal Position of People with Physical or Intellectual Disabilities). SCP, The Hague.

Diener E. \& Fujita F. (1995) Resources, personal strivings, and subjective well-being: a nomothetic and idiographic approach. Journal of Personality and Social Psychology 68, 926-35.

Dijker A., Van Alphen L., Bos A., Van den Borne B. \& Curfs L. (2011) Social integration of people with intellectual disability: insights from a social-psychological research program. Journal of Intellectual Disability Research 55, 885-94.

Dusseljee J. C. E., Rijken P. M., Cardol M., Curfs L. M. G. \& Groenewegen P. P. (2011) Participation in daytime activities among people with mild or moderate intellectual disability. Journal of Intellectual Disability Research 55, 4-18.

Duvdevany I. \& Arar E. (2004) Leisure activities, friendships, and quality of life of persons with intellectual disability: foster homes vs community residential settings. International Journal of Rehabilitation Research 27, 289-96.

Evenhuis H. M. (2011) Stand van zaken. Gezondheid van ouderen met verstandelijke handicaps (State of affairs. Health of older people with an intellectual disability). Nederlands Tijdschrift voor Geneeskunde 155, 1-7.

Evenhuis H. M., Theunissen M., Denkers I., Verschuure H. \& Kemme H. (2001) Prevalence of visual and hearing impairment in a Dutch institutionalized population with intellectual disability. Journal of Intellectual Disability Research 45, 457-64.

Finlay W. M. L. \& Lyons E. (2001) Methodological issues in interviewing and using self-report questionnaires with people with mental retardation. Psychological Assessment 13, 319-35.

Forrester-Jones R., Carpenter J., Coolen-Schrijner P., Cambridge P., Tate A., Beecham J. et al. (2006) The social networks of people with intellectual disability living in the community 12 years after resettlement from long-stay hospitals. Journal of Applied Research in Intellectual Disabilities 19, 285-95.

Frieswijk N., Buunk B. P., Steverink N. \& Slaets J. P. J. (2004) The effect of social comparison information on the life satisfaction of frail older persons. Psychology and Ageing 19, 183-90.

Janicki M. P., Dalton A. J., Henderson C. M. \& Davidson P.W. (1999) Mortality and morbidity among older adults with intellectual disability: health services considerations. Disability and Rehabilitation 21, 284-94.

Jansen D. E. M. C., Krol B., Groothoff J.W. \& Post D. (2004) People with an intellectual disability and their health problems: a review of comparative studies. Journal of Intellectual Disability Research 48, 93-102.

Kearney D. S. \& Healy O. (2011) Investigating the relationship between challenging behavior, co-morbid psychopathology and social skills in adults with moderate to severe intellectual disabilities in Ireland. Research in Developmental Disabilities 32, 1556-63.

Kirk R. (2007) Effect magnitude: a different focus. Journal of Statistical Planning and Inference 137, 1634-46.

Lloyd V., Gatherer A. \& Kalsy S. (2006) Conducting qualitative interview research with people with expressive language difficulties. Qualitative Health Research 16,

1386-405.

Losier G. F., Bourque P. E. \& Vallerand R. J. (1993) A motivational model of leisure participation in the elderly. Journal of Psychology 127, 153-70.

McCallion P. \& McCarron M. (2004) Ageing and intellectual disabilities: a review of recent literature. Current Opinion in Psychiatry 17, 349-52.

McCarron M. \& Lawlor B. A. (2003) Responding to the challenges of ageing and dementia in intellectual disability in Ireland. Aging and Mental Health 7, 413-17.

Ormel J., Lindenberg S., Steverink N. \& Verbrugge L. M. (1996) Subjective well-being and social production functions. Social Indicators Research 46, 61-90.

Rosenthal J. A. (1996) Qualitative descriptions of strength of association and effect size. Journal of Social Service Research 21, 37-59.

Schalock R. L., Borthwick-Duffy S. A., Bradley V. J., Buntinx W. H. E., Coulter D. L., Craig E. M. et al. (2010) Intellectual Disability: Definition, Classification, and Systems of Supports, 11th edn. AAID, Washington DC. 
Lehmann, B.A., Bos, A.E.R., Rijken, M., Cardol, M., Peters, G.J.Y., Kok, G., Curfs, L.M.G. Ageing with an intellectual disability: the impact of personal resources on well-being. Journal of Intellectual Disability Research: 2013, 57(11), 1068-1078

Smith J., Borchelt M., Maier H. \& Jopp D. (2002) Health and well-being in the young old and oldest old. Journal of Social Issues 58, 715-32.

Thorpe L., Davidson P. \& Janicki M. (2001) Healthy ageing - adults with intellectual disabilities: biobehavioural issues. Journal of Applied Research in Intellectual Disabilities 14, 218-28.

Van Alphen L. M., Dijker A. J. M., Borne H. H.W. \& Curfs L. M. G. (2009) The significance of neighbours: views and experiences of people with intellectual disability on neighbouring. Journal of Intellectual Disability Research 53, 745-57.

Van Schrojenstein Lantman-de Valk H. M. J., Metsemakers J. F. M., Haveman M. J. \& Crebolder H. F. J. M. (2000) Health problems in people with intellectual disability in general practice: a comparative study. Family Practice 17, 405-7.

Van Schrojenstein Lantman-de Valk H. M. J., Te Wierik M. J. M., Van den Akker M., Wullink M., Schellevis F. G., Dinant G. J. et al. (2004) Morbidity and health-care use in people with Intellectual Disability in general practice: first results of a survey in the Netherlands. Journal of Policy and Practice in Intellectual Disabilities 1, 107-9.

Zantinge E. M., van der Wilk E. A., van Wieren S. \& Schoemaker C. G. (2011) Gezond ouder worden in Nederland. Rijksinstituut voorVolksgezondheid en Milieu (RIVM) (Healthy ageing in the Netherlands. National Institute for Public Health and the Environment). Available at RIVM: http://rivm.nl/bibliotheek/rapporten/270462001.pdf (retrieved 1 November 2011).

\section{TABLES}

Table 1: Qualitative effect size labels used to identify relevant associations.

\begin{tabular}{llll}
\hline & Small/weak & Medium/moderate & Large/strong \\
\hline Odds ratio & $<0.67$ or $>1.5$ & $<0.4$ or $>1.5$ & $<0.25$ or $>4.0$ \\
$\eta^{2}$ & $>0.01$ & $>0.06$ & $>0.14$ \\
Cohen's $d$ & $>0.20$ & $>0.50$ & $>0.80$ \\
Pearson's $r$ & $>0.10$ & $>0.30$ & $>0.50$ \\
Cramèr's V & $>0.10$ & $>0.30$ & $>0.50$ \\
\hline
\end{tabular}


Lehmann, B.A., Bos, A.E.R., Rijken, M., Cardol, M., Peters, G.J.Y., Kok, G., Curfs, L.M.G. Ageing with an intellectual disability: the impact of personal resources on well-being. Journal of Intellectual Disability Research: 2013, 57(11), 1068-1078

Table 2 Absolute and relative frequency distributions of dichotomous variables measured in 2006 and 2010 for the total sample and for participants aged 50 and older

\begin{tabular}{|c|c|c|c|c|c|c|c|c|c|c|c|c|}
\hline & \multicolumn{6}{|c|}{ Total sample } & \multicolumn{6}{|c|}{$50+$ Participants } \\
\hline & \multicolumn{3}{|c|}{2006} & \multicolumn{3}{|c|}{2010} & \multicolumn{3}{|c|}{2006} & \multicolumn{3}{|c|}{2010} \\
\hline & $\begin{array}{l}\text { No } \\
n(\%)\end{array}$ & $\begin{array}{l}\text { Yes } \\
n(\%)\end{array}$ & Total & $\begin{array}{l}\text { No } \\
n(\%)\end{array}$ & $\begin{array}{l}\text { Yes } \\
n(\%)\end{array}$ & Total & $\begin{array}{l}\text { No } \\
n(\%)\end{array}$ & $\begin{array}{l}\text { Yes } \\
n(\%)\end{array}$ & Total & $\begin{array}{l}\text { No } \\
n(\%)\end{array}$ & $\begin{array}{l}\text { Yes } \\
n(\%)\end{array}$ & Total \\
\hline Physical health & $51(10)$ & $447(90)$ & 498 & $64(13)$ & $433(87)$ & 497 & $30(13)$ & $195(87)$ & 225 & 34 (I7) & $165(83)$ & 199 \\
\hline $\begin{array}{l}\text { Restricted } \\
\text { mobility }\end{array}$ & & & & $301(64)$ & $|7|$ (36) & 472 & & & & $126(67)$ & $63(33)$ & 189 \\
\hline Disabilities & $360(69)$ & $158(31)$ & 518 & & & & $159(70)$ & $67(30)$ & 226 & & & \\
\hline Visual & 451 (87) & $67(13)$ & 518 & & & & $19 \mid(85)$ & $35(15)$ & 226 & & & \\
\hline Auditory & 460 (89) & 58 (II) & 518 & & & & $192(85)$ & 34 (15) & 226 & & & \\
\hline Motor & 398 (77) & $120(23)$ & 518 & & & & I7I (76) & $55(24)$ & 226 & & & \\
\hline Communicative & $468(90)$ & $50(10)$ & 518 & & & & $210(93)$ & $16(7)$ & 226 & & & \\
\hline Autism & 459 (89) & 59 (II) & 518 & & & & $202(89)$ & 24 (II) & 226 & & & \\
\hline Behavioural & $450(87)$ & 68 (13) & 518 & & & & $200(88)$ & $26(12)$ & 226 & & & \\
\hline Psychiatric & $455(88)$ & $63(12)$ & 518 & & & & $191(85)$ & $35(15)$ & 226 & & & \\
\hline Addiction & 512 (99) & $6(1)$ & 518 & & & & $223(99)$ & $3(1)$ & 226 & & & \\
\hline Dementia & $513(99)$ & $5(I)$ & 518 & & & & $221(98)$ & $5(2)$ & 226 & & & \\
\hline Epilepsy & $438(85)$ & $80(15)$ & 518 & & & & $197(87)$ & $29(13)$ & 226 & $125(67)$ & 61 (33) & 186 \\
\hline Social satisfaction & $126(28)$ & $332(72)$ & 458 & 77 (17) & $379(83)$ & 456 & 57 (28) & 144 (72) & 201 & $25(14)$ & $153(86)$ & 178 \\
\hline Has a partner & $335(65)$ & 178 (35) & 513 & $320(64)$ & $177(36)$ & 497 & $160(69)$ & 71 (3I) & 231 & $|3|(66)$ & $69(35)$ & 200 \\
\hline $\begin{array}{l}\text { Partner also } \\
\text { has intellectual } \\
\text { disabilities }\end{array}$ & $29(18)$ & $130(82)$ & 159 & $32(19)$ & $136(8 I)$ & 168 & II (19) & $48(81)$ & 59 & $9(14)$ & $56(86)$ & 65 \\
\hline Has children & $485(95)$ & $28(5)$ & 513 & $480(96)$ & $19(4)$ & 499 & $218(94)$ & $13(6)$ & 231 & $193(96)$ & $8(4)$ & 201 \\
\hline $\begin{array}{l}\text { Satisfaction } \\
\text { with life }\end{array}$ & $47(9)$ & 451 (9I) & 498 & $51(10)$ & $439(90)$ & 490 & $18(9)$ & $176(91)$ & 194 & $14(7)$ & $182(93)$ & 196 \\
\hline Happiness & & & & $72(15)$ & $415(85)$ & 487 & & & & $30(15)$ & $167(85)$ & 197 \\
\hline Loneliness & $259(55)$ & $211(45)$ & 470 & $290(62)$ & $179(38)$ & 469 & $98(54)$ & $85(46)$ & 183 & $125(67)$ & 61 (33) & 186 \\
\hline $\begin{array}{l}\text { Lives } \\
\quad \text { independently }\end{array}$ & 51 (II) & 431 (89) & 482 & $56(12)$ & $429(88)$ & 485 & $26(14)$ & 157 (86) & 183 & $36(18)$ & $168(82)$ & 204 \\
\hline
\end{tabular}

Table 3 Descriptive statistics describing the continuous variables measured in 2010

\begin{tabular}{|c|c|c|c|c|c|c|c|c|}
\hline & \multicolumn{4}{|c|}{ Total sample } & \multicolumn{4}{|c|}{ 50+ Participants } \\
\hline & Mean & Median & Range & $\begin{array}{l}\text { Standard } \\
\text { deviation }\end{array}$ & Mean & Median & Range & $\begin{array}{l}\text { Standard } \\
\text { deviation }\end{array}$ \\
\hline Age & 46.73 & 46 & $17-86$ & 14.94 & 61.01 & 59 & $50-86$ & 8.38 \\
\hline Number of children & 0.08 & 0 & $0-4$ & 0.44 & 0.06 & 0 & $0-2$ & 0.33 \\
\hline Number of disabilities & 1.11 & 1 & $0-5$ & 1.03 & 1.16 & 1 & $0-4$ & 1.04 \\
\hline
\end{tabular}


Lehmann, B.A., Bos, A.E.R., Rijken, M., Cardol, M., Peters, G.J.Y., Kok, G., Curfs, L.M.G. Ageing with an intellectual disability: the impact of personal resources on well-being. Journal of Intellectual Disability Research: 2013, 57(11), 1068-1078

Table 4 Effect sizes and $P$-values for the cross-sectional bivariate analyses of the 2010 data to examine which variables are associated with age

\begin{tabular}{|c|c|c|c|}
\hline & \multicolumn{3}{|c|}{ Age } \\
\hline & \multicolumn{2}{|c|}{ Effect size* } & \multirow{2}{*}{$\begin{array}{l}P \\
0.034\end{array}$} \\
\hline Physical health & $\mathrm{S}$ & $d:-0.28$ & \\
\hline Restricted mobility & M & $d: \quad 0.58$ & 0.000 \\
\hline Disabilities: & & d: $\quad 0.00$ & 0.980 \\
\hline Visual & $S$ & $d: \quad 0.31$ & 0.017 \\
\hline Auditory & M & $d: 0.55$ & 0.000 \\
\hline Motor & & $d: \quad 0.02$ & 0.823 \\
\hline Communicative & $\mathrm{S}$ & $d:-0.32$ & 0.030 \\
\hline Autism & $\mathrm{S}$ & $d:-0.20$ & 0.036 \\
\hline Behavioural & & $d:-0.19$ & 0.142 \\
\hline Psychiatric & $\mathrm{S}$ & $d: \quad 0.30$ & 0.024 \\
\hline Addiction & & $d:-0.03$ & 0.951 \\
\hline Dementia & M & $d: \quad 0.64$ & 0.157 \\
\hline Epilepsy & & $d:-0.02$ & 0.838 \\
\hline Number of disabilities & & $r: \quad 0.04$ & 0.412 \\
\hline Social satisfaction & $\mathrm{S}$ & $d: \quad 0.30$ & 0.017 \\
\hline Has a partner & & $d:-0.06$ & 0.506 \\
\hline $\begin{array}{l}\text { Partner also has } \\
\text { intellectual disabilities }\end{array}$ & $\mathrm{S}$ & d: $\quad 0.24$ & 0.219 \\
\hline Number of children & & $r: \quad 0.00$ & 0.922 \\
\hline Leisure time autonomy & $S$ & $\eta^{2}: \quad 0.02$ & 0.007 \\
\hline Decision-making autonomy & $\mathrm{S}$ & $\eta^{2}: \quad 0.03$ & 0.002 \\
\hline Satisfaction with life & $\mathrm{S}$ & $d: 0.31$ & 0.034 \\
\hline Happiness & & $d: \quad 0.01$ & 0.946 \\
\hline Loneliness & $S$ & $d:-0.20$ & 0.036 \\
\hline Lives independently & $S$ & $d:-0.37$ & 0.009 \\
\hline
\end{tabular}

* S, small; $\mathrm{M}$, medium; L, large. 
Lehmann, B.A., Bos, A.E.R., Rijken, M., Cardol, M., Peters, G.J.Y., Kok, G., Curfs, L.M.G. Ageing with an intellectual disability: the impact of personal resources on well-being. Journal of Intellectual Disability Research: 2013, 57(11), 1068-1078

Table 5 Odds ratios and $P$-values for the longitudinal bivariate analyses to examine variable stability and identify predictors in 2006 of satisfaction with life, happiness and loneliness in 2010

\begin{tabular}{|c|c|c|c|c|c|c|c|c|c|c|c|c|}
\hline \multirow[b]{2}{*}{ Variables in 2006} & \multicolumn{3}{|c|}{$\begin{array}{c}\text { Variable stability } \\
\text { (association with self) }\end{array}$} & \multicolumn{3}{|c|}{$\begin{array}{l}\text { Loneliness } \\
\text { in } 2010\end{array}$} & \multicolumn{3}{|c|}{$\begin{array}{l}\text { Satisfaction with } \\
\text { life in } 2010\end{array}$} & \multicolumn{3}{|c|}{$\begin{array}{l}\text { Happiness } \\
\text { in } 2010\end{array}$} \\
\hline & & t size* & $P$ & & ct size* & $P$ & & t size* & $P$ & & ct size* & $P$ \\
\hline Physical health & $M$ & 0.35 & 0.035 & $S$ & 0.34 & 0.029 & & 0.75 & $0.786^{\dagger}$ & M & 3.60 & 0.019 \\
\hline Social satisfaction & $M$ & 2.50 & 0.018 & $S$ & 0.50 & 0.106 & $\mathrm{~S}$ & 0.41 & $0.393^{\dagger}$ & $S$ & 0.52 & $0.316^{t}$ \\
\hline Has a partner & & 1.33 & 0.664 & & 0.67 & 0.321 & $S$ & 1.89 & $0.426^{\dagger}$ & & 0.70 & 0.464 \\
\hline $\begin{array}{l}\text { Partner also has } \\
\text { intellectual disabilities }\end{array}$ & $\mathrm{S}$ & 1.50 & $1.000^{\ddagger}$ & $S$ & 0.33 & $0.265^{\dagger}$ & & 1.15 & $0.702^{\dagger}$ & $\mathrm{S}$ & 1.50 & $0.738^{+}$ \\
\hline Has children & & 1.00 & $1.000^{\ddagger}$ & L & 6.21 & $0.077^{\dagger}$ & & 1.04 & $0.534^{\dagger}$ & & 1.04 & $0.347^{\dagger}$ \\
\hline Satisfaction with life & & 1.14 & $1.000^{\ddagger}$ & $S$ & 0.36 & $0.136^{\dagger}$ & & & & $\mathrm{S}$ & 0.43 & 0.416 \\
\hline Loneliness & $S$ & 0.64 & 0.118 & & & & $M$ & 3.14 & $0.146^{t}$ & & 1.41 & 0.479 \\
\hline Lives independently & & 0.86 & $1.000^{\ddagger}$ & & 1.22 & 0.707 & & 1.21 & $0.171^{\dagger}$ & $\mathrm{L}$ & 0.24 & $0.142^{\dagger}$ \\
\hline
\end{tabular}

* S, small; M, medium; L, large.

${ }^{\dagger}$ In this $\chi^{2}$ analysis, one or more cells had an expected value of 5 or less. The $P$-value can therefore be inaccurate. However, all Fisher's exact tests had similar $P$-values (i.e. in all situations, either both $P$-values were significant or both were non-significant).

₹ Note that when using binomial distributions, $P$-values of $\mathrm{I}$ result when there is no association. 